\title{
Exact Outage Probability Caused by Multiple Nakagami Interferers with Arbitrary Parameters
}

\author{
Qiuyan Liu, Zhangdui Zhong, Bo Ai, Miao Wang \\ State Key Laboratory of Rail Traffic Control and Safety \\ Beijing JiaotongUniversity, Beijing, China, 100044 \\ E-mail: \{07120230, zhdzhong, boai, 05211045\}@ bjtu.edu.cn
}

\author{
César Briso-Rodríguez \\ Universidad Politécnica de Madrid \\ Madrid 28031, Spain \\ E-mail: cbriso@diac.upm.es
}

\begin{abstract}
In this paper, outage probability caused by multiple interferers in Nakagami- $m$ fading channels is studied. A novel method is proposed to derive the exact and closed form expressions of outage probability in the presence of multiple Nakagami independent cochannel interference. Unlike some previous conclusions, the method proposed in this paper is not only usable with various integer Nakagami fading parameters or average powers but also presents exact derivation of outage probability. To circumvent the difficulties, proper iteration functions are adopted by studying and integrating the definitions without numerical integration and residue calculation. The method generally deals with the cases that with or without minimum level constraint at the receiver for satisfactory reception. Finally, the exact expressions are compared with previous proposed approximated expressions and provide the understanding of the nature of interference.
\end{abstract}

\section{INTRODUCTION}

The development of wireless communication is just the history of interference. The interference analyses provide understandings of the nature of interference to develop the interference management and reduction techniques. Of all frequency non-selective fast fading models, Nakagami- $m$ distribution is the most versatile [1]. The interference analysis in Nakagami- $m$ fading channels is a general and essential problem in wireless communication. Outage probability is one of the most important criterion of interference performance. Here, we will be concerned with outage probability caused by interference in Nakagami- $m$ fading extensively.

Only a few papers, but approximated methods, have been proposed in previous work on the outage probability caused by arbitrary Nakagami interferers [2-6]. Abu-Dayya, Zhang, Tellamabura did notable work to derive the outage probability with direct numerical inversion of the characteristic function method (CHM). The basic problem commonly confronted was to deduce outage probability from characteristic function, including at least two fold integral for two inevitable processes:

1) Derive the inverse Fourier transformation of the characteristic function of SIR.

2) Calculate the cumulative density function of the inverse Fourier transformation.

Usually, the computation complex is too high to get an exact closed form expression with arbitrary parameters. Another method is proposed to get the approximated outage probability [7]. Reig solved the problem with approximate distribution of multiple Nakagami interferers. To the knowledge of the authors, no exact closed form expressions have been given in the literature. In this paper, we propose a novel method to derive the exact form expression of outage probability with multiple Nakagami interferers. In comparison with the methods in literatures, three advantages of the new method are obvious:

1) Closed and exact expressions of outage probability caused by multiple Nakagami interferers with arbitrary parameters are given.

2) The outage probability caused by $n+1$ interferers can be easily iterated from the conclusion of $n$ interferers.

3) Compared to the results with minimum level constraint, the outage probability without constraint can be easily derived by defining the receiver threshold as zero.

The remainder of this paper is organized as follows. Section II formalizes the derivation of outage probability. In Section III, the comparison and analyses based on different methods in some special cases are studied. The precisions of different methods are also discussed in Section III. Finally, extensive understandings of the nature of multiple Nakagami interferers and conclusions are drawn in Section IV.

\section{FORMULATION}

Outage probability is generally defined as the probability of unsatisfactory reception. In practice, two criterion are in common use. The first one is indicated to consider the interference only which is useful in the interference dominant conditions such as microcell and picocell network. However, the outage probability caused by coverage is too significant to be neglected when the cell size is large enough like macrocell network. Compared to the first definition theoretically, the second one is a modification with minimum signal power constraint. The mathematical definitions of outage probability is generally described as

$$
\begin{aligned}
& P_{\text {out }}=1-\int_{w}^{\infty} \int_{\lambda}^{\infty} f_{x_{0}, \operatorname{SIR}}(y, x) \mathrm{d} x \mathrm{~d} y \\
& =1-\int_{w}^{\infty} \int_{0}^{\lambda y} f_{x_{0}}(y) f_{\mathrm{I}}(x) \mathrm{d} x \mathrm{~d} y
\end{aligned}
$$

where $f_{x_{0}}(\cdot), f_{I}(\cdot)$ and $f_{\operatorname{SIR}}(\cdot)$ are the distribution functions of signal, aggregate interference and the ratio of signal-tointerference, respectively. The minimum signal level $w$ is more or less of the same magnitude order with local mean power, 
usually specified from $-110 \mathrm{dBm}$ to $-80 \mathrm{dBm}$. $\lambda$ is denoted as the SIR protection ratio. The second definition can be regarded as the first one when $w=0$. Obviously, the outage probability is obtained from a two fold integral.

In Nakagami channel, the power of signal and interference can be described as follows

$$
f(x ; m, \Omega)=\frac{m^{m}}{\Gamma(m) \Omega^{m}} x^{m-1} \exp \left(-\frac{m}{\Omega} x\right), \quad x>0
$$

where $m>0.5$ is Nakagami fading parameter; $\Omega$ denotes the local mean power; and $\Gamma(\cdot)$ is gamma function.

Let total interference be power addition of each interference under incoherent assumption. Substitute (2) into (1), the general expressions of outage probability with $n$ interferers is

$$
\begin{gathered}
P_{\text {out }}^{n}=1-\int_{w}^{\infty} f\left(x_{0} ; m_{0}, \Omega_{0}\right) \int_{0}^{x_{0} / \lambda} f\left(x_{1} ; m_{1}, \Omega_{1}\right) \\
\cdots \times \int_{0}^{x_{0} / \lambda-\sum_{i=1}^{n-1} x_{i}} f\left(x_{n} ; m_{n}, \Omega_{n}\right) \mathrm{d} x_{n} \cdots \mathrm{d} x_{1} \mathrm{~d} x_{0}
\end{gathered}
$$

where $x_{0}, m_{0}, \Omega_{0}$, and $x_{i}, m_{i}, \Omega_{i}, i \in[1, n]$ are the variables, fading parameters and average power of the signal and the $i^{t h}$ interference, respectively.

Note that more than one interferers can be combined as a single interferer if they have identical Nakagami fading parameters $m$ and average power $\Omega$ [3]. In the general case, let $m_{i} \neq m_{j}$ and $\Omega_{i} \neq \Omega_{j}$ for $i \neq j(i, j \in[1, n])$. In the intuitive view of definition, there appears to exist an analytical solution of outage probability with proper iterative functions. To guarantee the integrability, the definition in equation (3) is slightly modified with sufficient condition

$$
x_{1}=\left\{x_{i} \mid \frac{m_{i}}{\Omega_{i}}=\max _{j \in[1, n]}\left\{\frac{m_{j}}{\Omega_{j}}\right\}\right\}
$$

Define

$$
\begin{aligned}
& g_{i}=\int_{0}^{\kappa_{i-1}} f\left(x_{i} ; m_{i}, \Omega_{i}\right) \int_{0}^{\kappa_{i}} f\left(x_{i+1} ; m_{i+1}, \Omega_{i+1}\right) \\
& \cdots \times \int_{0}^{\kappa_{n}} f\left(x_{n} ; m_{n}, \Omega_{n}\right) \mathrm{d} x_{n} \cdots \mathrm{d} x_{i+1} \mathrm{~d} x_{i}
\end{aligned}
$$

with $\kappa_{1}=x_{0} / \lambda$ and $\kappa_{i}=x_{0} / \lambda-\sum_{j=1}^{i-1} x_{j}, i \in[2, n]$, then $\forall i \in[1, n]$, we have

$$
\exists g_{i}=\int_{0}^{\kappa_{i}} f\left(x_{i} ; m_{i}, \Omega_{i}\right) \times g_{i+1} \mathrm{~d} x_{i}
$$

Obviously, the outage probability with $i+1$ is easily derived only with one more iterative function. And when $i=0$, the corresponding connection probability is defined as the signal above the minimum level threshold

$$
g_{0}=\int_{w}^{\infty} f\left(x_{0} ; m_{0}, \Omega_{0}\right) \times g_{1} \mathrm{~d} x_{0} .
$$

Also, the outage probability caused by $n$ Nakagami interferers is given by

$$
P_{\text {out }}^{n}=1-g_{0} \text {. }
$$

with $\kappa_{i}=\kappa_{i-1}-x_{i-1}$. Then, the processes of choosing appropriate iterative functions and derivation of outage probability are listed as follows.

Step 1: The first fold integral is expressed by the incomplete gamma function [8].

$$
\begin{aligned}
& g_{n}=\int_{0}^{\kappa_{n}} f\left(x_{n} ; m_{n}, \Omega_{n}\right) \mathrm{d} x_{n} \\
& =1-\frac{\Gamma\left(m_{n}, \frac{m_{n}}{\Omega_{n}} \kappa_{n}\right)}{\Gamma\left(m_{n}\right)}
\end{aligned}
$$

where $\gamma(\cdot, \cdot)$ is the lower incomplete gamma function. Substitute equation (9) into (3), the outage probability is rewritten as

$$
\begin{gathered}
P_{\text {out }}^{n}=P_{\text {out }}^{n-1}+\int_{w}^{\infty} f\left(x_{0} ; m_{0}, \Omega_{0}\right) \cdots \\
\times \int_{0}^{\kappa_{n-1}} f\left(x_{n-1} ; m_{n-1}, \Omega_{n-1}\right) \frac{\Gamma\left(m_{n}, \frac{m_{n}}{\Omega_{n}} \kappa_{n}\right)}{\Gamma\left(m_{n}\right)} \mathrm{d} x_{n-1} \cdots \mathrm{d} x_{0}
\end{gathered}
$$

Obviously, the outage probability caused by $n$ interferers can easily iterated from the conclusion of $n-1$ interferers.

Step 2: The second fold integral is expanded as

$$
\begin{aligned}
& g_{n-1}=\int_{0}^{\kappa_{n-1}} f\left(x_{n-1} ; m_{n-1}, \Omega_{n-1}\right) \times g_{n} \mathrm{~d} x_{n-1} \quad \text { (11) } \\
= & \int_{0}^{\kappa_{-1}} \frac{m_{n-1}^{m_{n-1}} x_{n-1}^{m_{n-1}-1} \gamma\left(m_{n}, \frac{m_{n}}{\Omega_{n}} \kappa_{n}\right)}{\Omega_{n-1}^{m_{n-1}} \Gamma\left(m_{n-1}\right) \Gamma\left(m_{n}\right) \exp \left(\frac{m_{n-1}}{\Omega_{n-1}} x_{n-1}\right)} \mathrm{d} x_{n-1}
\end{aligned}
$$

The relevant integral is written as

$$
h_{n-1}=\int_{0}^{\kappa_{n-1}} x^{m} \exp (-\beta x) \gamma\left(s, c\left(\kappa_{n-1}-x\right)\right) \mathrm{d} x
$$

Theorem Let $m>0, \beta>0, s>0, u>0, c>0$, and $k_{m}=\int_{0}^{u} x^{m} \exp (-\beta x) \Gamma(s, c(u-x)) \mathrm{d} x . k_{m}$ is integrable and can be represented as closed form expression. Then,

$$
\begin{aligned}
& k_{m}=\sum_{i=1}^{m} A_{m, i} e^{-c u} \gamma(s+i,(\beta-c) u) \\
& +\sum_{i=1}^{m} B_{m, i} u^{i} e^{-\beta u}+C_{m} e^{-c u} \gamma(s,(\beta-c) u)+D_{m} e^{-\beta u}
\end{aligned}
$$

where $A_{m, i}, B_{m, i}, C_{m}$, and $D_{m}$ also represent the constants independent of the variable. The provision of the theorem is outlined in Appendix I.

Accordingly, $h_{n-1}$ is also integrable and can be rewritten as closed form expression

$$
h_{n-1}=\Gamma(s) \beta^{-m-1} \gamma\left(m+1, \beta \kappa_{n-1}\right)-k_{n-1}
$$

Step 3: Furthermore, we calculate the third fold integration $g_{n-2}$.

$$
g_{n-2}=\int_{0}^{\kappa_{n-2}} f\left(x_{n-2} ; m_{n-2}, \Omega_{n-2}\right) \times g_{n-1} \mathrm{~d} x_{n-2}
$$


The relevant integral is

$$
\begin{aligned}
& h_{n-2}=\int_{0}^{\kappa_{n-2}} x^{m} e^{-\beta x} h_{n-1} \mathrm{~d} x \\
& \Leftrightarrow \sum_{i=0}^{m} \int_{0}^{\kappa_{n-2}} A_{n-2, i} x^{m} e^{-\beta x} \gamma\left(s+i, c\left(\kappa_{n-2}-x\right)\right) \mathrm{d} x \\
& -\sum_{i=0}^{m} B_{n-2, i} \int_{0}^{\kappa_{n-2}} x^{m+i} e^{-\beta x} \mathrm{~d} x
\end{aligned}
$$

with constants $A_{n-2, i}$ and $B_{n-2, i}$.

The closed form expression of the first term is given by returning to step 2. And the second term is given by [9]

$$
\begin{aligned}
& \sum_{i=0}^{m} B_{n-2, i} \int_{0}^{\kappa_{n-2}} x^{m+i} \exp (-\beta x) \mathrm{d} x \\
& =\sum_{i=0}^{m} B_{n-2, i} \beta^{-m-i-1} \gamma\left(m+i+1, \beta \kappa_{n-2}\right)
\end{aligned}
$$

\section{Step 4:}

Then, we go on to solve the fourth fold integration.

$$
g_{n-3}=\int_{0}^{\kappa_{n-3}} f\left(x_{n-3} ; m_{n-3}, \Omega_{n-3}\right) \times g_{n-2} \mathrm{~d} x_{n-3}
$$

The relevant integral is written as

$$
\begin{aligned}
& h_{n-3}=\int_{0}^{\kappa_{n-3}} x^{m} e^{-\beta x} h_{n-2} \mathrm{~d} x \\
& \Leftrightarrow \sum_{i=0}^{m} \int_{0}^{\kappa_{n-3}} A_{n-3, i} x^{m} e^{-\beta x} \gamma\left(s+i, c\left(\kappa_{n-3}-x\right)\right) \mathrm{d} x \\
& -\sum_{i=0}^{m} B_{n-3, i} \int_{0}^{\kappa_{n-3}} x^{m+i} \exp (-\beta x) \mathrm{d} x
\end{aligned}
$$

Fortunately, equation (19) has the same structure of the kernel with equation (16). Return to step 3 iteratively until we get $g_{1}$. The iterative process is discovered finally.

\section{Step 5:}

Besides the multiple interferers are considered, the outage probability has to be integrated over the fading distribution of the signal in such circumstance with and without constraint. The system requires minimum level constraint when $w>0$, and vice versa.

$$
g_{0}=\int_{w}^{\infty} f\left(x_{0} ; m_{0}, \Omega_{0}\right) \times g_{1} \mathrm{~d} x_{0}
$$

The relevant integral is

$$
\begin{aligned}
& h_{0}=\int_{w}^{\infty} x^{m} e^{-\beta x} h_{1} \mathrm{~d} x \\
& \Leftrightarrow \sum_{i=0}^{m} \int_{w}^{\infty} A_{0, i} x^{m} \exp (-\beta x) \gamma(s+i, c x) \mathrm{d} x \\
& -\sum_{i=0}^{m} B_{0, i} \int_{\lambda}^{\infty} x^{m+i} \exp (-\beta x) \mathrm{d} x
\end{aligned}
$$

The first integral term can be obtained in the similar way in Appendix I. The second integral term is also given by [9]

$$
\begin{aligned}
& \sum_{i=0}^{m} B_{0, i} \int_{\lambda}^{\infty} x^{m+i} \exp (-\beta x) \mathrm{d} x \\
& =\sum_{i=0}^{m} B_{0, i} \beta^{-m-i-1} \Gamma(m+i+1, \beta \lambda)
\end{aligned}
$$

Note that equation (20) and (21) is integrable if $m_{1} / \Omega_{1}>$ $m_{i} / \Omega_{i}$, for $i \in(1, n]$, which is equivalent to the conclusion in [5].

\section{Step 6:}

Finally, the outage probability $P_{\text {out }}^{n}$ is given by Equation (8).

There are no approximations but closed expressions in each step above. Therefore, the general outage probability is inevitable to be exact and closed.

\section{COMPARISON AND CASES STUdiEd}

\section{A. Comparison With the Approximated Methods}

Through the application of the general method described above, one can derive the exact outage probability expressions for many different generalized fading channels of practical interest. Although there are several methods proposed to approximate the outage probability caused by multiple Nakagami interferers, all the approximated methods proposed can be categorized into Gamma distribution (GD) [2,7] and characteristic function methods (CHM)[3-6]. Fig. 1 shows the comparison between the exact expression (EE) method and the approximated Gamma distribution method derived in [7] and the CHM proposed in [3] . Note that both approximated methods have excellent agreement. To evaluate the precision of the approximated methods, the residue $\Delta$ of the approximated methods is defined as a criterion.

$$
\Delta=\left|P_{\text {out }}-\widehat{P}_{\text {out }}\right|
$$

where $P_{\text {out }}$ denotes the outage probability derived by the exact expressions mentioned above; $\widehat{P}_{\text {out }}$ represents the evaluated outage probability given by the approximated methods. Fig. 2 shows the residue between the exact expression and the approximated GD and CHM, respectively. The upper figure illustrates the outage probability versus SIR threshold $\lambda$ in linear unit, and the lower one figure out the residue delta of the approximated outage probability. Compared to the exact expression above, CHM is obviously more precise than GD. The residue of outage probability given by GD is about $10^{-2}$, while the residue of $\mathrm{CHM}$ is more or less $10^{-15}$. However, GD is absolutely exact only if all the interferers power are independent and identically distributed random variables for the virtue of GD.

Wireless communication system is usually modeled as a power decay system due to electromagnetic radiation. Here, an exponential power decay profile with decay factor $\delta$ is used to model the power imbalance, so that $\widetilde{\Omega}_{i}=\Omega_{i} e^{-\delta i}, i \in[0, n]$. Outage probability and approximated results obtained by the 


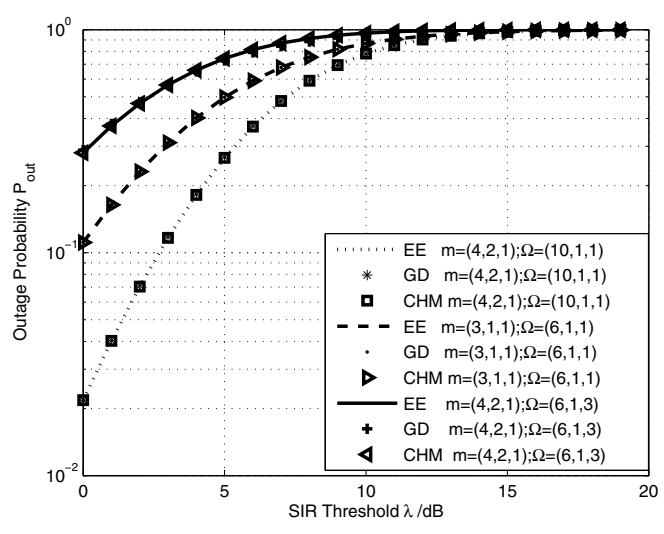

Fig. 1. Comparison between exact derivation and approximated methods

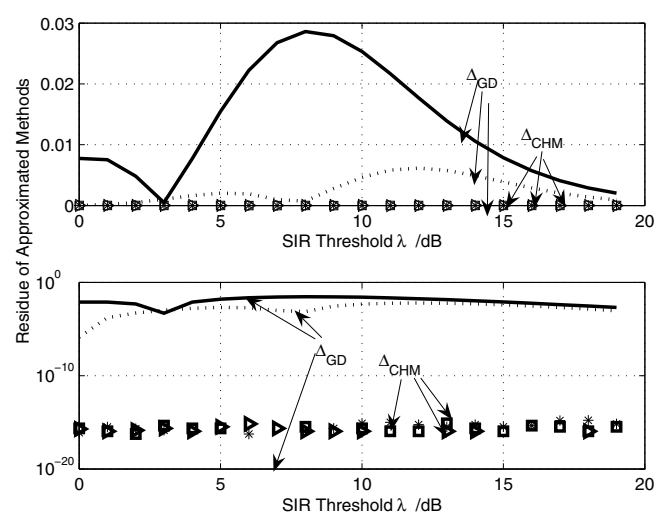

Fig. 2. The residue of approximated methods

EE, GD and CHM are considered in Fig. 3. Note that the approximated methods GD and CHM also have high precision. Fig. 4 illustrates the residues of GD and CHM deviating from EE in different conditions. Moreover, it is rigorous to for the GD method to be absolutely exact. In addition to the identical fading parameters and average power, the identical delay factors are also necessary. In addition, the complexity of $\mathrm{EE}, \mathrm{GD}$ and $\mathrm{CHM}$ are listed as follows. Obviously, the complexity of EE is of the same magnitude $O\left(\prod_{i=1}^{n} m_{n}\right)$ with $\mathrm{CHM}$ in [2]. Another CHM proposed by Zhang has excellent performance both in precision and complexity $O\left(n^{2}\right)$. So far, GD is the simplest method.

TABLE I

LAPLACE TRANSFORMS OF SEVERAL CHANNEL MODELS

\begin{tabular}{|l|l|l|l|}
\hline Methods & EE & GD & CHM \\
\hline Complexity & $O\left(\prod_{i=1}^{n} m_{n}\right)$ & $O(n)$ & $O\left(n^{2}\right)$ or $O\left(\prod_{i=1}^{n} m_{n}\right)$ \\
\hline
\end{tabular}

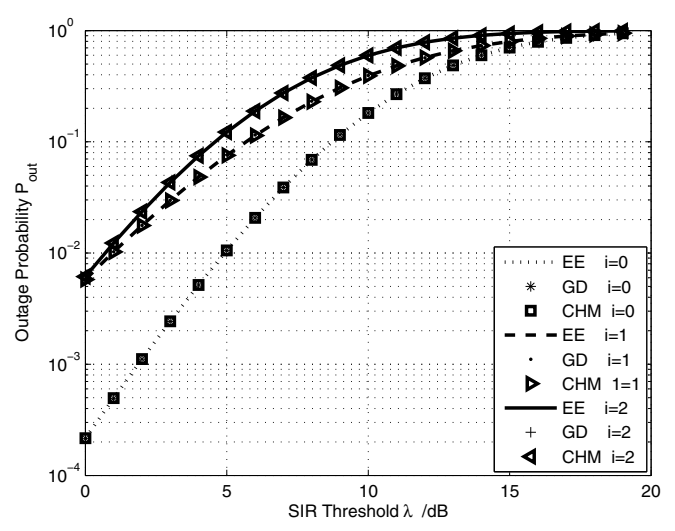

Fig. 3. Outage probability comparison with exponential power decay

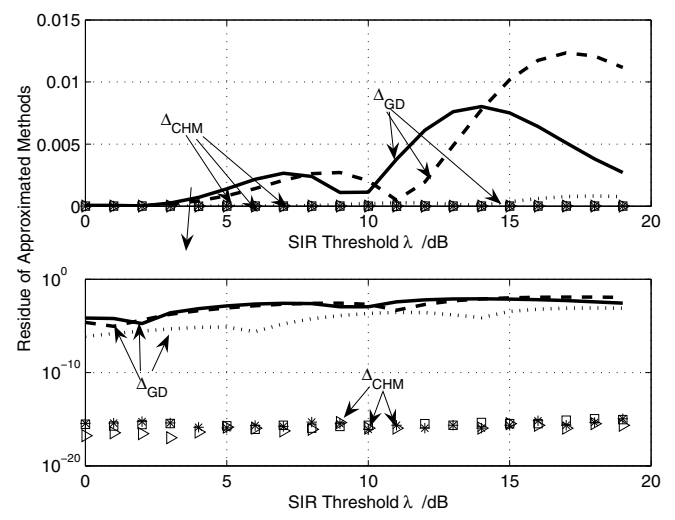

Fig. 4. Residue of approximated methods with exponential power decay

\section{B. Cases Studied}

In this section, we consider the special cases in linear coverage network illustrated in Fig. 5, which is representative in highway or railway systems. All the transmitters are located along the railways or highways with high power but no power control to ensure the safety and seamless coverage. The radius of the cell is $R \mathrm{~km}$, and the nearest two transmitters with the same frequency is $D \mathrm{~km}$ apart. Therefore, there are mainly two strong interferers even with line of sight (LOS) around the service cell. Extensive interference analyses can provide understandings of the nature of interference to develop the interference management and reduction techniques. For example, we consider different Nakagami fading parameters and average power with two interferers. Fig. 6 shows the exact outage probability for different network parameters. Note that it can usually satisfy the outage probability below $10^{-2}$ when SIR threshold is below $9 \mathrm{~dB}$ if reuse factor $D / R$ is more than 4 [10]. It is observed that as receiver SIR threshold increases, the outage probability degrades significantly with other parameters fixed. Compared curve a with d, more than $90 \%$ outage can be eliminated if SIR threshold is dropped from $15 \mathrm{~dB}$ to 9 $\mathrm{dB}$. In addition, outage probability is liable to be affected by the signal channel according to curve a, b and c. However, 
minimum signal level $w$ has little impact on outage probability, especially for the low $w$ range.

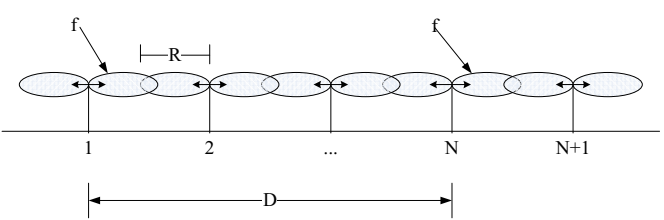

Fig. 5. Linear coverage networks

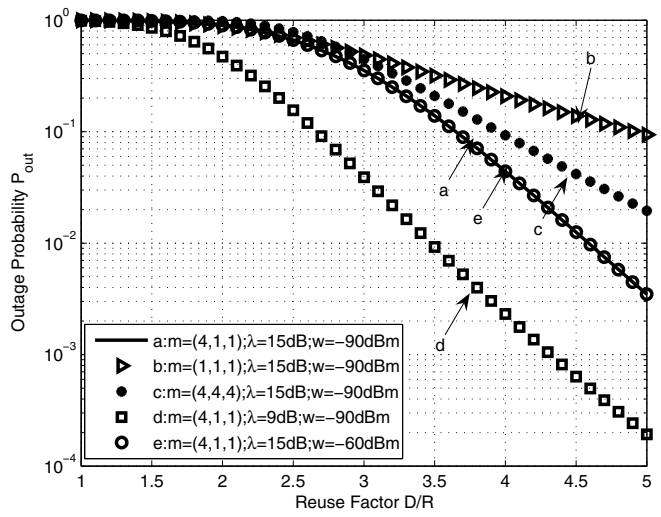

Fig. 6. The outage probability versus reuse factor

\section{CONCLUSION}

A novel method to derive the exact form expressions of multiple Nakagami interferers with arbitrary parameters has been investigated in this paper. More specially, with the consideration of such circumstances with or without minimum signal level constraints, exact and closed-form expressions have been derived for general situations. The final conclusions have been compared with the approximated Gamma distribution and characteristic function method to verify the precision and the residue of the approximated methods. Generally, compared with $\mathrm{EE}, \mathrm{CHM}$ is more precise than GD. GD has deviation from $\mathrm{EE}$ about $10^{-2}$, while $\mathrm{CHM}$ has derivation from $\mathrm{EE}$ about $10^{-15}$. The method obtained has also been applied in linear coverage network as special cases studied to provide understandings of the nature of interference.

\section{APPENDIX I}

To verify (13), the integration by parts is applied.

$$
\begin{aligned}
& k_{m}=\int_{0}^{u} x^{m} \exp (-\beta x) \Gamma(s, c(u-x)) \mathrm{d} x \\
& =-\left.\frac{x^{m}}{\beta} \exp (-\beta x) \Gamma(s, c(u-x))\right|_{0} ^{u} \\
& +\frac{1}{\beta} \int_{0}^{u} m x^{m-1} \Gamma(s, c(u-x)) \exp (-\beta x) \mathrm{d} x \\
& -\frac{c}{\beta} \int_{0}^{u} x^{m+s-1} \exp (-c(u-x)) \exp (-\beta x) \mathrm{d} x
\end{aligned}
$$

$$
\begin{aligned}
& =\frac{m}{\beta} k_{m-1}-\frac{c e^{-u c} \gamma(m+s,(\beta-c) u)}{(\beta-c)^{(m+s)} \beta}-\frac{u^{m} \Gamma(s) e^{-\beta u}}{\beta} \\
& =\frac{m}{\beta} k_{m-1}+A_{m} e^{-u c} \gamma(m+s,(\beta-c) u)+B_{m} u^{m} e^{-\beta u}
\end{aligned}
$$

Here, $k_{m}$ is an iterative function of $k_{m-1} . A_{m}$ and $B_{m}$ are the constants independent of $u$. When $m=1$, the initial term $k_{1}$ is

$$
\begin{aligned}
& k_{1}=\int_{0}^{u} x \exp (-\beta x) \Gamma(s, c(u-x)) \mathrm{d} x \\
& =-\frac{\Gamma(s) e^{-\beta u}}{\beta^{2}}-\frac{u \Gamma(s) e^{-\beta u}}{\beta} \\
& -\frac{c e^{-u c} \gamma(s,(\beta-c) u)}{\beta^{2}(\beta-c)^{s}}-\frac{c e^{-u c} \gamma(1+s,(\beta-c) u)}{(\beta-c)^{(1+s)} \beta} \\
& =A_{1} e^{-u c} \gamma(1+s,(\beta-c) u)+B_{1} u e^{-\beta u} \\
& +C_{1} e^{-u c} \gamma(s,(\beta-c) u)+D_{1} e^{-\beta u}
\end{aligned}
$$

Substitute (25) into (24),

$$
\begin{aligned}
& k_{m}=\sum_{i=1}^{m}\left(A_{i} e^{-u c} \gamma(i+s,(\beta-c) u)+B_{i} u^{i} e^{-\beta u}\right) \\
& +C_{m} e^{-u c} \gamma(s,(\beta-c) u)+D_{m} e^{-\beta u}
\end{aligned}
$$

where $A_{i}, B_{i}, i \in[1, m], C_{m}$ and $D_{m}$ are also the constants.

\section{ACKNOWLEDGMENT}

The authors would like to thank to the anonymous reviewers for their suggestions. And this work is supported by the joint state key program of the NSFC of China and the national railway ministry of China (Grant No. 6083001), and the programs of state key laboratory of traffic control and safety (RCS2008ZZ006 and RCS2008ZZ007).

\section{REFERENCES}

[1] M. Nakagami, "The m-distribution-A general formula of intensity distribution of rapid fading," Statistical Mehods of Radio Wave Propagation, W. G. Hoffman, Ed. Oxford, England: Pergamon 1960.

[2] A. A. Abu-Dayya, N. C. Beaulieu, "Outage probabilities of cellular mobile radio systems with multiple Nakagami interferers," IEEE Trans. Veh. Technol., vol.40, PP. 757-768, 1991.

[3] Q. T. Zhang, "Outage probability of cellular mobile radio in the presence of multiple Nakagami interferers with arbitrary fading parameters," IEEE Trans. Veh. Technol., vol. 44, no. 3, pp. 661-667, 1995.

[4] Q. T. Zhang, "Outage probability in cellular mobile radio due to Nakagami signal and interferers with arbitrary parameters," IEEE Trans. Veh. Technol., vol.45, pp. 364-372, 1996.

[5] C. Tellambura and V. K. Bhargava, "Outage probability analysis for the cellular mobile radio with multiple, arbitrary Nakagami interferers,' IEEE Vehicular Technology Conference, vol. 1, pp.384-388, 1995.

[6] C. Tellambura, "Cochannel interference computation for arbitrary Nakagami fading," IEEE Trans. Veh. Technol., vol.48, pp.487-489, 1999.

[7] J. Reig, N. Cardona, L. Rubio, "Approximation of outage probability on Nakagami fading channels with multiple interferers", Electronics Letters, vol. 36, no. 19, 2000.

[8] I. N. Bronshtein, and K. A. Semendyayev, Handbook of Mathematics, $4^{\text {th }}$ Edition, Springer-Verlag, Berlin, 2004.

[9] I. S. Grandshteyn and I. M. Ryzhik, Table of integrals, series, and products, $7^{\text {th }}$ Edition, London: Acadmic Press, 2007.

[10] 3GPP TS 45.005 v9.1.0, "3rd Generation Partnership Project; Technical Specification Group GSM/EDGE Radio Access Network: Radio transmission and reception", Release 9, Nov. 2009. 